\title{
Antecedentes de Expectativas de Carreira na Organização
}

\author{
Vinicius Carvalho de Vasconcellos ${ }^{1}$ \\ Elaine Rabelo Neiva \\ Universidade de Brasília, Brasília, DF, Brasil
}

\begin{abstract}
Resumo
Diante da instabilidade do mundo organizacional, a questão do futuro na carreira atrai cada vez mais a atenção dos trabalhadores. Neste sentido, este artigo analisou a influência de cinco variáveis antecedentes nas expectativas dos indivíduos sobre suas carreiras na atual organização. As variáveis antecedentes foram: sexo, tempo de organização do indivíduo, cargo ocupado, obtenção (ou não) de conquistas profissionais recentes e a participação (ou não) em programas de desenvolvimento de carreira. O estudo contou com três amostras independentes, oriundas de uma autarquia federal $(n=305)$, de uma empresa de economia mista $(n=265)$ e de um conjunto de organizações de distintos setores/segmentos da economia $(n=352)$. Expectativas de carreira foram aferidas pela Escala de Expectativas de Carreira na Organização. Os dados indicaram que indivíduos em cargos gerenciais, participantes de programas de desenvolvimento e com conquistas profissionais recentes detêm expectativas mais favoráveis do que os demais. O efeito das demais variáveis foi pequeno/inexistente. Na discussão, os resultados são analisados a luz das contribuições e limitações da pesquisa.
\end{abstract}

Palavras-chave: Expectativas, desenvolvimento profissional, organizações.

\section{Antecedents of Career Expectations in the Organization}

\begin{abstract}
Considering the instability of the organizational world, the question of future career is increasingly attracting the attention of workers. This paper analyzed the influence of five antecedent variables on the individuals' expectations about their careers in the current organization. The antecedent variables were: gender, respondent's organizational tenure, career position, obtainment of recent professional achievements and participation in career development programs. The study had three independent samples coming from a government agency $(n=305)$, from a mixed-capital corporation $(n=265)$ and from a set of organizations covering different sectors /segments of the economy $(n=352)$. The career expectations were measured by the Career Expectations in the Organization Scale. The data indicated that individuals in managerial positions, participants of career development programs and professionals with recent professional achievements hold better career expectations. The effects of the other variables were small or nonexistent. In the discussion, the results were analyzed considering the contributions and limitations of the research.
\end{abstract}

Keywords: Expectations, professional development, organizations.

Endereço para correspondência: Universidade de Brasília, Instituto de Psicologia, Departamento de Psicologia Social e do Trabalho, Campus Universitário Darcy Ribeiro - ICC Ala Sul, Brasília, DF, Brasil 70900-910. Caixa-postal: 70833030. E-mail: viniciuscarvalhodevasconcellos@gmail.com 


\section{Antecedentes de las Expectativas de Carrera en la Organización}

\section{Resumen}

Ante la inestabilidad del mundo organizacional, el futuro de la carrera atrae cada vez más la atención de los profesionales. Este trabajo analiza la influencia de cinco variables antecedentes sobre las expectativas de los profesionales acerca de su carrera en la organización actual. Las variables antecedentes fueron: sexo, el tiempo en la organización, cargo, la obtención (o no) de logros profesionales recientes y la participación (o no) en programas de desarrollo de carrera. El estudio tuvo tres muestras independientes procedentes de una agencia federal $(n=305)$, de una empresa de capital público/privado $(n=265)$ y de un conjunto de organizaciones de diferentes sectores/segmentos de la economía $(n=352)$. Expectativas de la carrera se midieron por la Escala de Expectativas de Carrera en la Organización. En general, los datos indican que las personas que ocupan cargos de liderazgo, participantes de programas de desarrollo de carrera y con logros profesionales recientes tienen expectativas más favorables que los otros. Los efectos de las otras variables fueron pequeños o inexistentes. En la discusión, los resultados son analizados a partir de las aportaciones y limitaciones de la investigación.

Palabras clave: Expectativas, desarrollo profesional, organizaciones.

A relação dos indivíduos com o futuro faz parte da subjetividade humana, estando presente nos mais diversos domínios sociais, como trabalho, vida familiar e comunidade, entre outros. No domínio do trabalho, foco desta pesquisa, projeções sobre o futuro profissional inserem-se nas discussões sobre carreira, entendida como sequência de experiências de trabalho no curso da vida de uma pessoa (Greenhaus, Callanan, \& Godshalk, 1999).

O futuro profissional configura-se como importante elemento na relação indivíduo-trabalho-organização, visto que perspectiva de crescimento na carreira surge entre as características mais valorizadas por trabalhadores em organizações (Tolfo, 2002) e que expectativas/percepções sobre o futuro na carreira relacionam-se com variáveis relevantes para a gestão, como atitudes no trabalho e intenções de desligamento (Chay \& Aryee, 1999; Chen, Ployhart, Cooper-Thomas, Anderson, \& Bliese, 2011; Stroh \& Reilly, 1997).

Partindo da relevância da matéria, este artigo trata especificamente das expectativas dos profissionais sobre o futuro de suas carreiras na sua organização atual. Assumiu-se neste trabalho expectativas de carreira na organização como as crenças dos indivíduos sobre sua futura trajetória profissional na atual organização (Vasconcellos, 2015), construto que engloba expectativas sobre a futura consecução de objetivos profissionais e a obtenção de sucesso na carreira, tocando em projeções sobre remuneração, prestígio e realização de tarefas interessantes e alinhadas às preferências pessoais. A título de esclarecimento, frisa-se que expectativas de carreira expressam projeções dos indivíduos sobre o que ocorrerá em seu futuro profissional (baseadas no cenário mais provável e realista), sendo diferentes, portanto, de aspirações de carreira, que refletem preferências, desejos e/ou objetivos dos profissionais.

O objetivo da pesquisa foi analisar em que medida as expectativas de carreira na organização variam em função de cinco variáveis antecedentes. Estas foram selecionadas por refletirem características pessoais e experiências de carreira recentes que comportam, segundo a literatura, potencial para influenciar as crenças dos indivíduos sobre seu futuro profissional. As variáveis pesquisadas foram: sexo, o tempo do profissional na organização, cargo ocupado (gerencial ou não gerencial), a obtenção de conquistas profissionais recentes e a participação recente em programas de desenvolvimento de carreira. Ao submeter tais variáveis ao teste empírico, o pre- 
sente estudo busca gerar informações úteis para os pesquisadores da área e para a formulação de políticas/práticas de recursos humanos $(\mathrm{RH})$ vinculadas ao desenvolvimento de carreira.

Foram utilizadas três amostras independentes, de modo que foi possível analisar até que ponto os resultados mantinham-se consistentes em diferentes contextos. Respeitando as proposições da Nova Estatística (Cumming, 2012), as análises estatísticas não se restringiram à significância estatística e valorizaram a estimação dos tamanhos dos efeitos e o cálculo de intervalos de confiança.

\section{Revisão de Literatura e Hipóteses da Pesquisa}

A formação de expectativas pode ser estudada a partir de diversos ângulos. Para a seleção das variáveis antecedentes, o presente estudo recorreu inicialmente à teoria das expectativas de estado (Correll \& Ridgeway, 2006). Esta estabelece que, em situações de grupo (o ambiente de trabalho, por exemplo), características pessoais socialmente relevantes como sexo, raça e idade influenciam as expectativas de desempenho de si mesmo e dos demais membros do grupo. Os autores atestam que, em função de estereótipos ou de crenças enraizadas na cultura, forjam-se expectativas de que indivíduos/grupos com certas características apresentarão maior desempenho que outros, sendo tais expectativas absorvidas até mesmo pelos indivíduos desvalorizados, em um processo que normalmente desemboca em profecias autorrealizadas. Indivíduos/grupos com expectativas mais positivas tendem a ter mais chances e condições de desempenhar um bom trabalho, levando-os gradualmente a posições de maior status. No presente estudo, as variáveis sexo e tempo de organização são analisadas a partir deste pano de fundo.

Outra fonte teórica importante para o presente estudo trata do impacto das experiências de realizações passadas/atuais na formação de expectativas de futuro (Bandura, 1977). Essas realizações fazem emergir nos indivíduos a percepção de domínio, sendo fundamental para a formação da autoeficácia. Considerando essa dinâmica no contexto do desenvolvimento profissional, cumpre avaliar até que ponto realizações e experiências positivas na carreira (por exemplo, ser promovido e ocupar um cargo gerencial) podem influenciar as expectativas de carreira. Nessa linha, este estudo ocupa-se de avaliar o impacto do cargo ocupado, da obtenção de conquistas profissionais recentes e da participação em programas de desenvolvimento de carreira nas expectativas de carreira.

As duas últimas variáveis investigadas, obtenção de conquistas profissionais recentes e participação em programas de desenvolvimento de carreira, foram aferidas apenas na terceira amostra (coletada posteriormente), fruto do processo de amadurecimento da pesquisa. Estas fornecem informações importantes na análise da evolução na carreira e do atual grau de investimento do indivíduo no futuro profissional, com possíveis implicações para a formação de expectativas de carreira na organização. As próximas subseções apresentam a literatura que trata da relação das cinco variáveis antecedentes com as expectativas de carreira e derivam as hipóteses testadas posteriormente no artigo.

\section{Sexo do Profissional e Expectativas de Carreira na Organização}

A teoria das expectativas de estado pontua o sexo como uma das características pessoais mais importantes na formação de expectativas de desempenho. Em diversos países, crenças culturais seculares apontam os homens como sendo melhores que as mulheres na realização da maioria das tarefas/trabalhos (Correll \& Ridgeway, 2006), cenário que tende a prejudicar a evolução de carreira das mulheres. De fato, há evidências de que as mulheres ainda estão sub-representadas em posições de liderança, o que sugere a existência de um teto de vidro, metáfora empregada pela literatura para designar barreiras invisíveis para a ascensão profissional de mulheres (Cook \& Glass, 2014; Olidi, Parejo, \& Padilla, 2013). Entre as possíveis causas para o teto de vidro estão: (a) persistência de estereótipos de gênero e discriminação; (b) escolha, por parte 
das mulheres, de profissões menos valorizadas socialmente e/ou de cargos menos elevados, por exemplo, para ter mais tempo com família; (c) exclusão de atividades sociais (fora do trabalho) que favorecem a formação da rede profissional de contatos (Barnett, 2004; Cook \& Glass, 2014; Olidi et al., 2013).

Nas últimas décadas, em contraste à influência do fenômeno teto de vidro, o aumento de escolaridade das mulheres e a redução do tempo despendido por elas em tarefas domésticas (Barnett, 2004) sugerem maior equilíbrio na evolução de carreira de homens e mulheres. Neste cenário de possível transformação social, esta pesquisa investiga, na realidade nacional, em que medida as expectativas de carreira diferem em função do sexo do(a) profissional.

Pesquisas pautadas em variáveis genéricas de percepções de oportunidades futuras e otimismo na carreira são inconsistentes sobre o tópico. Há estudos que relataram médias mais elevadas para os homens (Coetzee, 2012), que não acusaram diferença entre os sexos (Stroh \& Reilly, 1997) e que assinalaram percepções mais positivas em mulheres (Treadway, Breland, Adams, Duke, \& Willians, 2010). Todavia, ao focalizar apenas expectativas sobre recompensas, salários e promoções, outro quadro emerge. Gibson e Lawrence (2010), ao revisar a temática, sinalizaram que investigações empíricas convergem ao apontar que mulheres exibem expectativas mais baixas sobre recompensas formais. E os dados obtidos por esses mesmos autores corroboraram esses estudos prévios. Em consonância, O’Neill, Stanley, e O'Reilly (2011) evidenciaram que as expectativas de salário de topo da carreira entre mulheres eram inferiores às expectativas dos homens.

Apesar do dissenso da literatura em abordagens mais genéricas, assumiu-se a hipótese de que os homens suplantam as mulheres nas expectativas de carreira, haja vista que o enfoque adotado na presente pesquisa inclui expectativas de remuneração e recompensas, matéria na qual a literatura aponta mais nitidamente médias maiores entre os homens. Assim, estipula-se que:

Hipótese 1 - Homens apresentam expectativas de carreira na organização maiores do que as mulheres.

\section{Tempo de Organização e Expectativas de Carreira}

A relação entre tempo de organização e expectativas de carreira pode ser analisada de várias formas. Por exemplo, é plausível supor que a passagem do tempo permite aos profissionais ganhar experiência, compreender melhor a dinâmica organizacional e desenvolver as habilidades requeridas pelo trabalho $(\mathrm{Ng}$ \& Feldman, 2011). Nessa mesma linha, meta-análise recente ( $\mathrm{Ng} \&$ Feldman, 2010) apontou relação positiva entre tempo de organização e desempenho. Partindo da premissa que desempenho mais elevado e experiência no ambiente de trabalho propiciam vantagens na evolução de carreira, é possível conceber a relação positiva entre tempo de organização e expectativas de carreira na organização. É provável ainda que profissionais com baixas expectativas de carreira não permaneçam muito tempo na organização, o que também favoreceria a correlação positiva entre as variáveis.

Contudo, essa relação parece ser mais intrincada. Buckley, Fedor, Veres, Wiese e Carraher (1998) explicitam que recém-admitidos tendem a criar expectativas elevadas sobre sua atuação no trabalho, especialmente porque a curta vivência no ambiente laboral os induz a minimizar ou ignorar as dificuldades existentes. Em paralelo, seguindo as ponderações da teoria das expectativas de estado, existem estereótipos sobre os profissionais recém-admitidos que podem influenciar as expectativas de desempenho. Espera-se que eles trabalhem mais duro para conseguir seu espaço na organização, que não apresentem vícios institucionais e que estejam mais abertos à mudança e à aprendizagem. Essas crenças tendem a gerar boas oportunidades para os recém-admitidos, consequentemente incrementando seu otimismo sobre o futuro.

Em discussão teórica, Taormina (1997) fornece um quadro mais completo de como as expectativas podem variar ao longo do tempo de organização. $\mathrm{O}$ autor propõe que os primeiros anos de organização geralmente despertam níveis elevados de expectativas, não apenas em função dos elementos citados acima, mas também por causa da novidade e excitação trazidas pela nova 
fase na carreira. Depois de adquirir mais clareza sobre a cultura e as práticas organizacionais, os profissionais enfrentam um choque de realidade e dividem-se então em três grupos. O primeiro é composto por uma minoria com grande talento, o que permite a continuidade das expectativas favoráveis de carreira. O segundo, formado por grande contingente de profissionais de desempenho mediano, reduz suas expectativas de carreira após o choque de realidade e o mantém em um platô por longo período. O terceiro, com indivíduos de desempenho limitado, diminui sucessivamente as expectativas de carreira, sendo grande a possibilidade de desligamento.

Neste enquadre, os primeiros anos de organização se caracterizariam pela associação negativa das variáveis (pouco tempo de organização e expectativas elevadas). Nos períodos seguintes, a maioria dos profissionais apresentaria um platô de expectativas, o que sinaliza ausência de relação entre as variáveis (a passagem do tempo não aumentaria nem diminuiria expectativas). Esse padrão é consistente com o efeito "lua de mel" (período inicial de avaliação positiva do trabalho) e "ressaca" (decréscimo nessa avaliação ao longo do tempo), sugerido pela literatura de atitudes no trabalho (Judge \& Kammeyer-Mueller, 2012).

Investigações empíricas desvelaram relação negativa, porém fraca, entre tempo de organização e medidas genéricas sobre projeções de futuro na carreira na organização (Chay \& Aryee, 1999; Chen et al., 2011; Vasconcellos \& Neiva, 2014). Outros estudos identificaram correlações na mesma direção ao medirem expectativas de níveis salariais (Gibson \& Lawrence, 2010) e a confiança dos indivíduos em conseguir um futuro favorável na organização (Jans, 1989), contudo, com magnitude maior (cerca de -0,30). Além da correlação, Jans (1989) testou a diferença de médias de grupos em distintos estágios de tempo de organização e os dados alinharam-se ao efeito "lua de mel" e "ressaca".

Com efeito, na literatura de carreira e de comportamento organizacional, tempo de organização pode ser aferido como variável métrica em anos (Gibson \& Lawrence, 2010) ou como variável não métrica que indica a fase da carreira na atual organização (Cohen, 1991; Jans, 1989; Ng \& Feldman, 2011; Wright \& Bonett, 2002). No último caso, as fronteiras que balizam os estágios variam entre as pesquisas, porém, o uso de intervalos de cinco anos está entre as opções mais frequentes (Ng \& Feldman, 2011; Wright $\&$ Bonett, 2002).

Considerando essas duas possibilidades, o presente estudo, além da análise correlacional, investiga se as médias de expectativas de carreira diferem entre grupos com distintos tempos de organização no cenário nacional. Em conjunto, os dados sugerem relação negativa, malgrado a divergência sobre o tamanho do efeito. Assim, conjectura-se que:

Hipótese $2 a$ - Profissionais no estágio inicial de carreira na organização detêm expectativas de carreira na organização maiores do que os demais.

Hipótese $2 b$ - Tempo de organização (como variável métrica) correlaciona-se negativamente com expectativas de carreira na organização.

\section{Cargo Ocupado e Expectativas de Carreira na Organização}

O cargo ocupado pelo profissional figura como outra variável que descreve a atual situação de carreira. Esta pesquisa comparou cargos gerenciais e não gerenciais, haja vista que tal distinção existe em praticamente todas as organizações. Assume-se aqui cargo gerencial como posição formal de liderança nas organizações, destinada em geral a profissionais que coordenam atividades em equipes de trabalho. Não obstante a variedade de rótulos para tal atividade, gerente será o termo genérico utilizado para designá-la nessa pesquisa.

O trabalho gerencial costuma exigir habilidades de negociação, facilidade para estabelecer relacionamentos interpessoais, capacidade de monitorar grande volume de informações (inclusive confidenciais) e perícia para tomar boas decisões (Mintzberg, 1990). Em complemento, cargos gerenciais habitualmente demandam elevadas cargas de trabalho, sendo comuns os conflitos com a vida pessoal (Lima, Carvalho, \& Tanure, 2012). 
Como a atuação gerencial pode influenciar expectativas de carreira? Em artigo teórico, Kahneman e Lovallo (1993) sustentam que boa parte dos gestores constrói uma autoimagem que supõe alto controle sobre indivíduos e eventos. Tal suposição de controle pode se desdobrar para o próprio futuro profissional e engendrar expectativas positivas sobre este último. Evidências nesse sentido procedem do campo experimental. Ao manipular entre os participantes os papéis de gerente e subordinado, Fast, Gruenfeld, Sivanathan e Galinsky (2009) destacam que a experiência de poder nos gerentes levou à ilusão de controle pessoal e, sequencialmente, aumentou seu grau de otimismo sobre os resultados do trabalho.

Outras razões para presumir níveis mais altos de expectativas de carreira em gerentes podem ser aventadas. Gerentes, em geral, detêm acesso privilegiado a informações e grande rede de contatos, elementos que permitem antecipar mudanças organizacionais e tomar decisões de carreira mais fundamentadas, melhorando suas expectativas na carreira. Adicionalmente, como a designação para cargo gerencial costuma ser interpretada como atestado de qualificação, competência e/ou importância dos promovidos (De Souza, 2002), gerentes tendem a apresentar autoavaliação profissional positiva, facilitando a emergência de crenças sobre a obtenção/manutenção de boas posições e recompensas no futuro na carreira.

Pesquisas empíricas convergem em sinalizar tal relação. Veloso et al. (2011) relataram que executivos percebem mais oportunidades de crescimento na organização do que não executivos. De Souza (2002) desvelou que o número de promoções recebidas no passado opera como preditor de expectativas de promoção no futuro e que profissionais que desejavam, mas que não lograram cargos gerenciais e promoções, reduzem suas expectativas de promoções futuras. Similarmente, há relação forte e positiva do atual nível hierárquico na organização com expectativas de salário no futuro (Gibson \& Lawrence, 2010) e com percepção de novas opções e possibilidades de carreira no futuro da organização (Treadway et al., 2010). Assim, formula-se a hipótese de que:
Hipótese 3 - Gerentes apresentam expectativas de carreira na organização maiores do que não gerentes.

\section{Conquistas e Desenvolvimento Profissional Recentes e Expectativas de Carreira}

Esta subseção aborda a possível influência das conquistas profissionais e da participação em programas de desenvolvimento de carreira nas expectativas dos indivíduos. A primeira variável denota, em grandes linhas, a evolução ou estagnação profissional, de modo que indivíduos que obtiveram recentemente conquistas profissionais tendem a perceber a carreira em crescimento, ao passo que indivíduos que não lograram tais conquistas tendem a julgar que sua carreira atingiu um platô ou que está em retrocesso.

Um ponto importante é indagar o que, de fato, constitui uma conquista profissional. $\mathrm{Na}$ verdade, há amplo espectro de respostas possíveis, a depender do que cada indivíduo valoriza e almeja para si. Aumento salarial, mudança de atividade, reconhecimento formal por suas contribuições e a obtenção de determinado cargo (promoção) são algumas das possibilidades. Percebe-se então que essa variável não se confunde com o cargo ocupado, uma vez que tornar-se gerente não constitui a única forma de conquista na carreira e que mesmo gerentes podem se sentir estagnados caso não tenham avanços recentes. Diante desse quadro, a opção nesta pesquisa foi respeitar a diversidade de significados das conquistas profissionais e deixar os respondentes livres para interpretar o termo, de modo que foi solicitado apenas que eles informassem se obtiveram ou não conquistas profissionais recentes na organização.

Dados empíricos sugerem a importância das conquistas profissionais na formulação de expectativas de carreira. Chen et al. (2011), fundamentados em desdobramentos da teoria dos prospectos (Kahneman, 1999), identificaram que mudanças recentes na satisfação com o trabalho predizem expectativas sobre a carreira e o ambiente de trabalho. Isto é, para além do atual patamar de satisfação, a mudança (melhoria ou piora) produz efeito nas expectativas. Propõe-se 
aqui testar essa dinâmica com mudanças na carreira ao invés de mudanças na satisfação no trabalho. Seguindo tais indicações, estabelece-se que:

Hipótese 4 - Indivíduos com conquistas profissionais recentes exibem expectativas de carreira na organização maiores que os demais.

Outra variável antecedente testada nessa pesquisa trata da participação recente em programas de desenvolvimento de carreira. Entre os programas de desenvolvimento de carreira mais comuns nas organizações estão mentoria, coaching, rodízio técnico, sucessão gerencial e suporte para planejamento de carreira. Essas iniciativas estão inseridas dentro de um plano maior, a saber, a gestão de carreira. Ao longo deste processo, os indivíduos obtêm feedback sobre suas carreiras, desenvolvem insights e adquirem conhecimento sobre o ambiente, formulando metas e estratégias para sua vida profissional (Greenhaus et al., 1999).

Parece plausível supor que os participantes desses programas detenham maior autoeficácia e estejam mais aptos e confiantes na condução de suas carreiras, apresentando assim expectativas mais favoráveis. Pesquisas empíricas reportam que profissionais de empresas que adotam tais programas percebem mais oportunidades de conseguir atividades desafiadoras (Eby, Allen, \& Brinley, 2005) e de crescer na carreira no futuro (Veloso et al., 2011). Essas duas pesquisas, contudo, aferiram se a organização do profissional ofertava tais práticas e não se o profissional, de fato, participava delas. A presente investigação avalia a relação da participação recente dos profissionais nessas práticas com suas expectativas de carreira. Baseado na literatura pregressa, conjectura-se que:

Hipótese 5 - Profissionais que participam ou participaram recentemente de programas de desenvolvimento de carreira exibem expectativas de carreira na organização maiores que os outros profissionais.

\section{Método}

\section{Participantes}

$\mathrm{O}$ estudo contou com três amostras independentes. Na amostra A $(n=305)$, coletada em uma autarquia federal, $74 \%$ eram homens e $70 \%$ não atuavam em cargo gerencial. A idade média foi 44,04 anos $(D P=9,50)$. O tempo médio de carreira na organização foi 13,36 anos $(D P=9,91)$, com esta distribuição: até cinco anos (28\%), entre seis e 10 anos (20\%) e 11 anos ou mais (52\%).

A amostra B $(n=265)$ provém de uma empresa de economia mista. Entre os respondentes, 75\% eram homens e $88 \%$ não atuavam em cargo gerencial. O questionário respeitou o padrão das pesquisas da organização de aferir idade e tempo na organização por meio de categorias. Por idade, informa-se que o grupo mais numeroso foi o de $35 \mathrm{a}$ 49 anos (40\%), seguido do grupo com até 34 anos (32\%) e do grupo com 50 anos ou mais (28\%). Por tempo de organização, a distribuição foi: até cinco anos (35\%), entre seis e 10 anos (37\%) e 11 anos ou mais $(28 \%)$.

Na amostra $C(n=352)$, a idade média foi de 42,26 anos $(D P=12,57), 51 \%$ eram homens e 58\% não exerciam cargo gerencial. Os respondentes trabalhavam em várias organizações, incluindo distintos setores/segmentos da economia. Contudo, a maioria (55\%) atuava na iniciativa privada. $\mathrm{O}$ tempo de organização médio foi de 8,44 anos $(D P=8,42)$, com a seguinte divisão: até cinco anos (52\%), entre seis e 10 anos (21\%) e 11 anos ou mais $(27 \%)$.

As amostras A e B foram coletadas no último trimestre de 2013. A amostra C foi coletada em setembro de 2014, como forma de reavaliar os resultados obtidos nas amostras A e B e examinar novas variáveis. De fato, as variáveis conquistas profissionais e participação recente em programas de desenvolvimento de carreira não foram testadas nas amostras A e B, sendo incluídas apenas na coleta de dados da amostra $\mathrm{C}$.

Nas três amostras, todos os indivíduos detinham escolaridade igual ou maior que o nível médio. Nas amostras A e B verificou-se que as proporções dos grupos foram similares àquelas encontradas na organização inteira. O tamanho de cada uma das três amostras superou o mínimo requerido para obter poder de $80 \%$ nos testes paramétricos e correlações, considerando $\alpha=0,05$ e expectativa de efeito pequeno para moderado (como $d=0,35, r=0,20$ e $\eta^{2}=0,04$ ). 


\section{Instrumentos}

O questionário continha itens sobre idade, tempo de organização, cargo ocupado (gerencial ou não gerencial) e sexo do respondente. Contava ainda com uma questão que perguntava se o profissional tinha obtido alguma conquista de carreira significativa na organização nos últimos dois anos (resposta dicotômica: sim ou não). Havia outra questão que indagava se o respondente fazia atualmente parte ou se tinha participado, nos últimos dois anos, de programas de desenvolvimento de carreira (resposta dicotômica: sim ou não). Em caso positivo, o respondente deveria citar o programa (exemplos indicados: rodízio técnico, coaching, mentoria, aconselhamento de carreira, cursos de alto impacto na carreira, entre outros). A análise dos programas citados confirmou a validade do item.

O questionário incluiu também a Escala de Expectativas de Carreira na Organização (EECO), instrumento validado por Vasconcellos (2015). Esta escala contém originalmente dois fatores: Conquistas Profissionais e Relação Carreira-Vida Pessoal. O primeiro fator agrupa conquistas estritamente dentro do ambiente de trabalho, incluindo projeções sobre remuneração, prestígio entre pares, consecução de objetivos profissionais e realização de atividades interessantes e alinhadas às preferências individuais. O segundo fator versa sobre expectativas de obtenção de uma relação favorável entre carreira/trabalho e vida pessoal/privada, abarcando projeções sobre o equilíbrio entre essas esferas e a compatibilidade entre necessidades pessoais e demandas de trabalho/carreira.

Em função do processo de negociação com as organizações, o fator Relação Carreira-Vida Pessoal não foi utilizado na coleta de dados das amostras A e B. Na amostra C, a escala foi aplicada com os dois fatores. Contudo, como as hipóteses do presente artigo sustentam-se em estudos anteriores voltados para elementos abordados pelo fator Conquistas Profissionais, optou-se por privilegiar a análise deste fator também nesta amostra. $\mathrm{Na}$ EECO, os participantes assinalam na escala a probabilidade da situação positiva descrita no item ocorrer cinco anos à frente (1=nada pro- vável até $7=$ extremamente provável). Seus nove itens aludem à futura situação de carreira do indivíduo naquela organização. Seguem alguns exemplos: "Atingirei meus objetivos de carreira estabelecidos para esse período", "Serei um profissional bem-sucedido na minha carreira" e "Minha remuneração será compatível com minhas expectativas de salário para esse período". Os escores de alfa de Cronbach foram 0,94 (amostras A e B) e 0,95 (amostra C).

\section{Procedimentos}

Nas amostras A e B, os respondentes foram selecionados aleatoriamente de uma lista de profissionais de cada organização. $\mathrm{Na}$ amostra C, uma consultoria especializada foi contratada para recrutar participantes e estes receberam um incentivo de cerca de $\mathrm{R} \$ 15$ reais para participar. Os participantes de todas as amostras receberam um correio eletrônico com informações sobre o estudo, instruções, contato do pesquisador e link de acesso ao questionário. $\mathrm{O}$ questionário foi respondido de forma individual, voluntária e confidencial.

\section{Análises de Dados}

Não houve outliers e os casos omissos estavam distribuídos assistematicamente. Na EECO, o escore consolidado da variável expressa a média dos itens. Os programas SPSS 19 e Statistica 8 foram utilizados para calcular correlações e realizar os testes estatísticos. Recorreu-se à Cohen (1992) para interpretar o tamanho dos efeitos.

\section{Resultados}

Inicialmente, todas as variáveis seriam submetidas a testes paramétricos. Contudo, houve diferenças consideráveis no tamanho dos grupos nas variáveis sexo e cargo ocupado das amostras A e B e na variável participação em programas de desenvolvimento de carreira da amostra C (apenas 20\% dos participantes responderam sim), o que pode prejudicar a precisão desses testes (Field, 2009). Optou-se então, nesses casos, pelo teste não paramétrico de Mann-Whitney. Nas demais variáveis, os grupos, além exibir 
igualdade de variância, registraram tamanhos razoavelmente equilibrados (Field, 2009), situação que favorece testes via análise de variância (ANOVA). A distribuição dos respondentes pelos grupos em cada variável antecedente está explicitada na seção de método, faltando apenas registrar que $51 \%$ dos respondentes da amostra $\mathrm{C}$ afirmaram que vivenciaram conquistas recentes na carreira.

\section{Tabela 1}

Expectativas de Carreira na Organização e sua Relação com as Variáveis Antecedentes do Estudo

\begin{tabular}{|c|c|c|c|c|c|c|c|c|c|}
\hline $\begin{array}{l}\text { Variável } \\
\text { Antecedente }\end{array}$ & Amostra & Grupos & Med. & Média & $D P$ & $\mathrm{IC}_{(95 \%)}$ & $\begin{array}{c}\text { Estatística } \\
\qquad(g l)^{\mathrm{a}}\end{array}$ & $p$ & Efeito $^{b}$ \\
\hline \multirow{6}{*}{ Sexo } & \multirow{2}{*}{ A } & Feminino & 4,78 & 4,58 & 1,31 & {$[4,26-4,89]$} & \multirow{2}{*}{$U=6860,50$} & \multirow{2}{*}{0,18} & \multirow{2}{*}{$\begin{array}{l}d=-0,21 \\
r=-0,08\end{array}$} \\
\hline & & Masculino & 4,56 & 4,30 & 1,31 & {$[4,12-4,47]$} & & & \\
\hline & \multirow{2}{*}{ B } & Feminino & 4,11 & 3,94 & 1,64 & {$[3,53-4,34]$} & \multirow{2}{*}{$U=5252,00$} & \multirow{2}{*}{0,03} & \multirow{2}{*}{$\begin{array}{l}d=0,33 \\
r=0,14\end{array}$} \\
\hline & & Masculino & 4,56 & 4,44 & 1,50 & {$[4,23-4,65]$} & & & \\
\hline & \multirow{2}{*}{$\mathrm{C}$} & Feminino & 4,67 & 4,37 & 1,51 & {$[4,14-4,60]$} & \multirow{2}{*}{$\begin{array}{c}F(1,350) \\
\quad=7,21\end{array}$} & \multirow{2}{*}{0,01} & \multirow{2}{*}{$\begin{array}{c}\eta_{\mathrm{p}}^{2}=0,02 \\
d=0,29 \\
r=0,14\end{array}$} \\
\hline & & Masculino & 5,11 & 4,78 & 1,32 & {$[4,58-4,97]$} & & & \\
\hline \multirow{9}{*}{$\begin{array}{l}\text { Tempo de } \\
\text { organização }\end{array}$} & \multirow{3}{*}{ A } & Até 5 anos & 4,78 & 4,48 & 1,34 & {$[4,18-4,77]$} & \multirow{3}{*}{$\begin{array}{c}F(2,282) \\
\quad=0,29\end{array}$} & \multirow{3}{*}{0,75} & \multirow{3}{*}{$\begin{array}{c}\eta_{\mathrm{p}}^{2}=0,00 \\
r=0,05\end{array}$} \\
\hline & & 6 a 10 anos & 4,50 & 4,35 & 1,42 & {$[3,97-4,74]$} & & & \\
\hline & & 11 anos ou mais & 4,67 & 4,35 & 1,25 & {$[4,14-4,55]$} & & & \\
\hline & \multirow{3}{*}{ B } & Até 5 anos & 4,56 & 4,45 & 1,42 & {$[4,16-4,75]$} & \multirow{3}{*}{$\begin{array}{c}F(2,257) \\
\quad=1,85\end{array}$} & \multirow{3}{*}{0,16} & \multirow{3}{*}{$\begin{array}{c}\eta_{\mathrm{p}}{ }^{2}=0,01 \\
r=0,12\end{array}$} \\
\hline & & 6 a 10 anos & 4,22 & 4,08 & 1,64 & {$[3,75-4,41]$} & & & \\
\hline & & 11 anos ou mais & 4,89 & 4,47 & 1,55 & {$[4,10-4,83]$} & & & \\
\hline & \multirow{3}{*}{$\mathrm{C}$} & Até 5 anos & 5,00 & 4,57 & 1,42 & {$[4,36-4,78]$} & \multirow{3}{*}{$\begin{array}{c}F(2,348) \\
=0,71\end{array}$} & \multirow{3}{*}{0,49} & \multirow{3}{*}{$\begin{array}{c}\eta_{\mathrm{p}}{ }^{2}=0,00 \\
r=0,06\end{array}$} \\
\hline & & 6 a 10 anos & 5,06 & 4,74 & 1,36 & {$[4,42-5,05]$} & & & \\
\hline & & 11 anos ou mais & 4,67 & 4,48 & 1,52 & {$[4,17-4,79]$} & & & \\
\hline \multirow{6}{*}{ Cargo } & \multirow[t]{2}{*}{ A } & Não Gerencial & 4,33 & 4,11 & 1,40 & {$[3,91-4,30]$} & \multirow[t]{2}{*}{$U=5507,50$} & \multirow{2}{*}{0,00} & \multirow{2}{*}{$\begin{array}{l}d=0,69 \\
r=0,30\end{array}$} \\
\hline & & Gerencial & 5,11 & 4,98 & 0,81 & {$[4,81-5,16]$} & & & \\
\hline & $\mathrm{B}$ & Não Gerencial & 4,33 & 4,22 & 1,55 & {$[4,02-4,42]$} & & 001 & $d=0,48$ \\
\hline & $\boldsymbol{D}$ & Gerencial & 5,11 & 4,95 & 1,37 & {$[4,45-5,45]$} & $0-2010,00$ & 0,01 & $r=0,15$ \\
\hline & $C_{-1}$ & Não Gerencial & 4,56 & 4,23 & 1,47 & {$[4,02-4,43]$} & $F(1,350)$ & 0 & $\eta_{\mathrm{p}}^{2}=0,08$ \\
\hline & $C$ & Gerencial & 5,44 & 5,06 & 1,23 & {$[4,86-5,26]$} & $=31,51$ & 0,00 & $\begin{array}{l}a-0,01 \\
r=0,29\end{array}$ \\
\hline Conquistas & $\mathrm{C}$ & Não & 4,06 & 4,01 & 1,51 & {$[3,77-4,25]$} & $F(1,330)$ & 0,00 & $\begin{array}{c}\eta_{\mathrm{p}}{ }^{2}=0,13 \\
d=0,76\end{array}$ \\
\hline na carreira ${ }^{\mathrm{c}}$ & & Sim & 5,22 & 5,03 & 1,18 & {$[4,86-5,21]$} & & & $r=0,36$ \\
\hline Desenvolvi- & $\mathrm{C}$ & Não & 4,78 & 4,43 & 1,45 & {$[4,26-4,60]$} & 50 & 00 & $d=0,54$ \\
\hline carreira $^{\mathrm{d}}$ & & Sim & 5,44 & 5,19 & 1,17 & {$[4,91-5,47]$} & & & $r=0,22$ \\
\hline
\end{tabular}

Nota. Med.=Mediana. $\mathrm{IC}_{(95 \%)}=$ Intervalo de Confiança de $95 \%$ da média.

a Estatísticas dos testes Mann-Whitney $(U)$ e análise de variância $(F)$. Os graus de liberdade estão entre parênteses.

${ }^{\text {b }}$ Tamanho do efeito da diferença entre grupos. Foi utilizada a forma não enviesada de cálculo de $d$ (Cumming, 2012). Para one-way ANOVAs: $\eta_{\mathrm{p}}^{2}=\eta^{2}$.

c Percepção do respondente sobre obtenção de conquistas na carreira nos últimos dois anos.

${ }^{\mathrm{d}}$ Participação em práticas de desenvolvimento de carreira nos últimos dois anos. 
A Tabela 1 explicita os diferentes patamares de expectativas de carreira na organização em função das variáveis antecedentes, além de reportar os testes de hipóteses. Como informado anteriormente, as variáveis conquistas recentes na carreira e participação recente em programas de desenvolvimento de carreira só foram aferidas na amostra C.

A hipótese 1 não foi suportada pela amostra A, que apontou expectativas mais elevadas nas mulheres, com efeito fraco e não significativo (Tabela 1). As amostras $\mathrm{B}$ e $\mathrm{C}$ assinalaram efeitos ainda fracos, porém ligeiramente maiores, na direção oposta e com significância estatística limítrofe, sinalizando apoio parcial para a hipótese 1. No geral, as amostras revelaram dados discrepantes, padrão que é perfeitamente possível considerando que os respondentes de cada amostra podem vivenciar realidades organizacionais bastante distintas. Por outro lado, de modo coerente com as proposições da nova estatística (Cumming, 2012), ressalta-se que, independente da direção dos efeitos, a magnitude desses últimos foi relativamente pequena no conjunto das amostras. Informa-se adicionalmente que a análise isolada dos itens sobre expectativas de recompensas e remuneração (indicados por estudos anteriores como mais favoráveis nos homens) não evidenciou diferenças representativas entre homens e mulheres.

Quanto ao tempo de organização, a segmentação expressa na Tabela 1 respeitou a proposta de $\mathrm{Ng}$ e Feldman (2011). Nas três amostras, as diferenças entre as médias dos grupos não foram significativas e os efeitos foram fracos. Logo, a hipótese 2a (trabalhadores no estágio inicial de carreira na organização detêm expectativas maiores do que os demais) não obteve suporte empírico. A hipótese $2 b$, que indicava relação negativa entre tempo de organização (como variável métrica) e expectativas de carreira, não se sustentou na amostra A $(r=-0,02, p=0,68)$ nem na amostra C $(r=-0,04$, $p=0,43)$. A correlação de Pearson não foi calculada na amostra $\mathrm{B}$, pois o tempo de organização foi aferido, como citado na seção sobre método, apenas por categorias e não como variável métrica.

Por outro lado, as três amostras suportaram a hipótese 3: gerentes explicitaram expectativas de carreira na organização maiores do que não gerentes, sendo o tamanho do efeito maior na amos- tra A, extraída de uma autarquia. Na amostra A, o tamanho do efeito foi moderado para forte; nas amostras B e C, moderado.

Profissionais que vivenciaram conquistas recentes na carreira exibiram expectativas maiores do que os demais profissionais em suporte à hipótese 4 (Tabela 1). A diferença foi estatisticamente significativa e o efeito de moderado para forte. Profissionais que participam ou participaram recentemente de programas de desenvolvimento de carreira revelaram expectativas maiores do que os outros profissionais (sustentando a hipótese 5). A diferença foi estatisticamente significativa e o efeito moderado.

\section{Discussão}

Os resultados evidenciaram efeitos dessemelhantes das variáveis antecedentes nas expectativas de carreira na organização. Expressando características pessoais que não podem alteradas pela gestão, as duas primeiras variáveis (sexo e tempo de organização) exibiram efeitos fracos nas expectativas de carreira. Em contrapartida, as demais variáveis antecedentes influenciaram mais claramente a forma com a qual os indivíduos visualizam seu futuro profissional.

A relação do sexo do respondente com as expectativas de carreira é controversa e inconsistente na literatura. $\mathrm{O}$ resultado do presente estudo sugere que, malgrado o fenômeno do teto de vidro citado na literatura (Cook \& Glass, 2014; Olidi et al., 2013), as expectativas de carreira não diferem substancialmente entre homens e mulheres, notadamente quando se observa o tamanho dos efeitos reportados. É possível que atual discussão na sociedade e nas organizações sobre equidade de gênero talvez esteja finalmente rendendo frutos. Por outro lado, cumpre atentar para a possível existência de variáveis moderadoras nessa relação, isto é, cuja presença/ausência, alterem a magnitude ou direção do efeito. Assim, o setor econômico (primário, secundário e terciário), a natureza da organização (pública, privada, de economia mista ou não governamental), o segmento de mercado (mineração, óleo e gás, varejo, saúde, entre outros) e principalmente as práticas de gestão e de recursos humanos de cada organização podem condicionar a existência de 
diferenças nas expectativas de carreira em função do sexo. Recomenda-se assim cautela na interpretação dos dados.

No caso de tempo de organização, os dados filiam-se aos estudos que descrevem ou propõem relação fraca/inexistente entre essas variáveis e expectativas de carreira na organização (Chay \& Aryee, 1999; Chen et al., 2011; Vasconcellos \& Neiva, 2014). Nessa linha, se há fatores que tendem a elevar expectativas nos recém- admitidos (como a percepção de futuro ilimitado e a "lua de mel" com a organização), existem igualmente elementos que favorecem as expectativas dos mais antigos na organização (maior experiência, alto desempenho e rede de contatos ampliada). No cenário contemporâneo, as aposentadorias são mais tardias, o que desafia as áreas de RH na temática da gestão de carreira. Esta pesquisa indica que expectativas favoráveis de carreira não se limitam aos recém-admitidos.

De sua parte, o cargo exerceu considerável efeito nas expectativas em todas as amostras. Nas análises empreendidas, gerentes obtiveram médias mais elevadas que não gerentes, conforme estudos prévios (De Souza, 2002; Gibson \& Lawrence, 2010; Veloso et al., 2011). Gerentes tendem a apresentar autoavaliação profissional positiva, acesso privilegiado a informações e grande rede de contatos, o que pode influir na formação de expectativas mais favoráveis.

A obtenção de conquistas recentes na carreira influenciou as expectativas de carreira na organização. Esse resultado complementa a pesquisa de Chen et al. (2011), que identificou que melhorias recentes na satisfação com o trabalho aumentam expectativas de carreira. $\mathrm{O}$ presente estudo sinalizou que conquistas recentes especificamente na carreira parecem atuar na mesma direção. Por sua vez, participantes em programas de desenvolvimento de carreira detêm expectativas de carreira mais positivas. Esse resultado era esperado, posto que tais programas tendem a qualificar seus participantes e a abrir novos horizontes no futuro profissional.

Cabe alertar que essas três últimas variáveis antecedentes, além de exibirem maior impacto nas expectativas de carreira, estão parcialmente sobrepostas. Gerentes tendem a participar mais de programas de desenvolvimento de carreira e a obter conquistas profissionais mais significativas que não gerentes. Contudo, uma vez que uma variável não esgota as demais, análises individuais, como as realizadas neste estudo, ainda são úteis. Seria pertinente ainda executar uma ANOVA fatorial testando conjuntamente essas variáveis antecedentes e suas interações, todavia, o tamanho amostral em cada célula impossibilitou tal análise.

Ao contrário de sexo e tempo de organização, essas três últimas variáveis antecedentes ainda compartilham uma característica comum. Elas distinguem profissionais com experiências recentes de carreira positivas/valorizadas daqueles que não gozam de tal situação. E os primeiros sempre apresentaram expectativas sobre o futuro de carreira mais elevadas, o que pode ser parcialmente explicado em função do incremento de autoeficácia (Bandura, 1977). É possível integrar os resultados dessas três variáveis a partir da interpretação de que há uma tendência entre esses profissionais de pensar o futuro de carreira como expansão de suas realizações/experiências profissionais recentes. Por exemplo, gerentes, sob a influência do atual sucesso profissional, estariam mais inclinados a extrapolar para o futuro o bom momento no presente. Cabe considerar que as expectativas de carreira foram aferidas a partir de projeções sobre remuneração, prestígio e consecução de objetivos profissionais, aspectos que esses profissionais provavelmente avaliam de forma positiva no presente.

Esta pesquisa contribui para a literatura, primeiramente, ao analisar em que medida os resultados citados na literatura internacional vigoram no contexto nacional. Cumpre ressaltar também que o estudo aborda e analisa tópico relevante na gestão de RH, qual seja, até que ponto profissionais com distintos perfis (homem/mulher, recém-admitidos/experientes, gerentes/não gerentes) diferem quanto às suas expectativas de carreira na organização. Ao se deparar com diferenças robustas e consistentes, como no caso dos gerentes/não gerentes, a gestão de RH deve ponderar sobre a necessidade de direcionar esforços em prol do grupo com menores expectativas. Nesta linha, a oferta de programas de desenvolvimento de carreira para não gerentes surge como caminho promissor, como sugerem os resultados aqui relatados. Esta é uma forma bastante interessante de integrar, na prática, os dados revelados 
pela pesquisa. Registra-se que todas as análises foram repassadas para as organizações pesquisadas individualmente (amostras A e B) como insumo para suas práticas de gestão.

Sublinha-se como limitações da pesquisa a impossibilidade de derivar relações causais, a utilização do autorrelato e o corte transversal da pesquisa. Sobre os participantes, embora as amostras A e B tenham sido geradas de listas aleatórias das organizações, a amostra $\mathrm{C}$ não passou por esse tipo de controle. Outra limitação refere-se à ausência, na pesquisa, de variáveis que podem exercer papel significativo nas expectativas de carreira, tais como traços de personalidade, desempenho no trabalho, políticas/ práticas de $\mathrm{RH}$ e expectativas sobre o futuro da organização. Investigações futuras devem considerar o efeito dessas variáveis nas expectativas de carreira, assim como avaliar se tempo de organização (variável com efeito pequeno/nulo) atua como moderadores dessas relações.

Por fim, se o futuro na carreira está entre as características mais valorizadas por trabalhadores em organizações (Tolfo, 2002), tal assunto não pode ser preterido. Mesmo que contrariem as hipóteses iniciais, análises sobre o tema podem subsidiar as organizações com informações úteis para a gestão de recursos humanos. A presente pesquisa deu um passo nessa direção. Espera-se que tanto a academia quanto as organizações invistam cada vez mais em estudos sobre desenvolvimento e expectativas de carreira.

\section{Referências}

Bandura, A. (1977). Self-efficacy: Toward a unifying theory of behavioral change. Psychological Review, 84, 191-215.

Barnett, R. (2004). Women and work: Where are we, where did we come from, and where are we going. Journal of Social Issues, 60(4), 667-674. doi:10.1111/j.0022-4537.2004.00378.x

Buckley, M., Fedor, D., Veres, J., Wiese, D., \& Carraher, S. (1998). Investigating newcomer expectations and job-related outcomes. Journal of Applied Psychology, 83, 452-461.

Chay, Y., \& Aryee, S. (1999). Potential moderating influence of career growth opportunities on careerist orientation and work attitudes: Evidence of the protean career era in Singapore. Journal of Organizational Behavior, 20(5), 613-623. doi:10.1002/ (SICI) 1099-1379(199909)20:5<613::AIDJOB979>3.0.CO;2-A

Chen, G., Ployhart, R., Cooper-Thomas, H., Anderson, N., \& Bliese, P. (2011). The power of momentum: A new model of dynamic relationships between job satisfaction change and turnover intentions. Academy of Management Journal, 54, 159-181. doi:10.5465/AMJ.2011.59215089

Coetzee, M. (2012). Distance learning students' graduateness as predictors of their job satisfaction and optimism about future career prospects. Journal of Social Sciences, 33(3), 305-313. Retrieved from http://uir.unisa.ac.za/ handle/10500/12045

Cohen, A. (1991). Career stage as a moderator of the relationships between organizational commitment and its outcomes: A meta-analysis. Journal of Occupational Psychology, 64(3), 253268. doi:10.1111/j.2044-8325.1991.tb00558.x

Cohen, J. (1992). A power primer. Psychological Bulletin, 112(1), 155-159. doi:10.1037/00332909.112.1.155

Cook, A., \& Glass, C. (2014). Women and top leadership positions: Towards an institutional analysis. Gender, Work \& Organization, 21(1), 91-103. doi:10.1111/gwao.12018

Correll, S. J., \& Ridgeway, C. L. (2006). Expectation states theory. In J. DeLamater (Ed.). Handbook of Social Psychology (pp. 29-51). New York: Springer.

Cumming, G. (2012). Understanding the new statistics: Effect sizes, confidence intervals, and meta-analysis. New York: Routledge.

De Souza, G. (2002). A study of the Influence of Promotions on Promotion Satisfaction and Expectations of Future Promotions among Managers. Human Resource Development Quaterly, 13(3), 325-340. doi:10.1002/hrdq.1034

Eby, L. T., Allen, T. D., \& Brinley, A. (2005). A cross-level investigation of the relationship between career management practices and career-related attitudes. Group and Organization Management, 30, 565-596. doi:10.1177/1059601104269118

Fast, N., Gruenfeld, D., Sivanathan, N., \& Galinsky, A. (2009). Illusory control a generative force behind power's far-reaching effects. Psychological Science, 20(4), 502-508. doi: 10.1111/j.1467-9280.2009.02311.x 
Field, A. (2009). Discovering statistics using SPSS. Thousand Oaks, CA: Sage.

Gibson, D., \& Lawrence, B. (2010). Women's and men's career referents: How gender composition and comparison level shape career expectations. $\mathrm{Or}$ ganization Science, 21, 1159-1175. doi:10.1287/ orsc. 1090.0508

Greenhaus, J., Callanan, G., \& Godshalk, V. (1999). Career management. Orlando, FL: Harcourt.

Jans, N. (1989). Organizational commitment, career factors and career/life stage. Journal of Organizational Behavior, 10(3), 247-266. doi:10.1002/ job.4030100305

Judge, T., \& Kammeyer-Mueller, J. (2012). Job attitudes. Annual Review of Psychology, 63, 341-367. doi:10.1146/annurev-psych-120710-100511

Kahneman, D. (1999). Objective happiness. In D. Kahneman, E. Diener, \& N. Schwartz (Eds.), Well-being: The foundations of hedonic psychology (pp. 3-25). New York: Sage.

Kahneman, D., \& Lovallo, D. (1993). Timid choices and bold forecasts: A cognitive perspective on risk taking. Management Science, 39(1), 17-31. doi:10.1287/mnsc.39.1.17

Lima, G., Carvalho, A., Neto, \& Tanure, B. (2012). Executivos jovens e seniores no topo da carreira: Conflitos e complementaridades. Revista Eletrônica de Administração, 18, 63-96. doi:10.1590/ S1413-23112012000100003

Mintzberg, H. (1990). The manager's job: Folklore and fact. Harvard Business Review, 68, 163-176. doi:10.1.1.568.7222

Ng, T., \& Feldman, D. (2010). Organizational tenure and job performance. Journal of Management, 36(5), 1220-1250. doi: $10.1177 / 0149206309359809$

Ng, T., \& Feldman, D. (2011). Affective organizational commitment and citizenship behavior: Linear and non-linear moderating effects of organizational tenure. Journal of Vocational Behavior, 79(2), 528-537. doi:10.1016/j.jvb.2011.03.006

O’Neill, O., Stanley, L., \& O’Reilly, C. (2011). Disaffected Pollyannas: The influence of positive affect on salary expectations, turnover, and long-term satisfaction. Journal of Occupational and Organizational Psychology, 84, 599-617. doi:10.1348/096317910X500801

Olidi, C., Parejo, S., \& Padilla, S. (2013). ¿Entre obstáculos anda el camino? Trayectoria y mujer directiva. Revista Psicologia: Organizações e
Trabalho, 13, 75-88. Recuperado em http://pepsic.bvsalud.org/pdf/rpot/v13n1/v13n1a07.pdf

Stroh, L., \& Reilly, A. (1997). Rekindling organizational loyalty: The role of career mobility. Journal of Career Development, 24, 39-54. doi:10.1023/A:1025034504937

Taormina, R. (1997). Organizational socialization: A multidomain, continuous process model. International Journal of Selection and Assessment, 5(1), 29-47. doi:10.1111/1468-2389.00043

Tolfo, S. (2002). Carreira profissional e seus movimentos: Revendo conceitos e formas de gestão. Revista: Psicologia, Organizações e Trabalho, 2(2), 39-63. Recuperado em https://periodicos. ufsc.br/index.php/rpot/article/view/6847

Treadway, D., Breland, J., Adams, G., Duke, A., \& Willians, L. (2010). The interactive effects of political skill and future time perspective on career and community networking behavior. Social Networks, 32, 138-147. doi:10.1016/j.socnet.2009.09.004

Vasconcellos, V. (2015). Antecedentes e consequentes de expectativas de carreira e de futuro organizacional (Tese de doutorado, Universidade de Brasília, DF, Brasil). Recuperado em repositorio.unb. br/handle/10482/18178

Vasconcellos, V., \& Neiva, E. (2014). Avaliação de futuro profissional e sua relação com bem-estar no trabalho e intenção de desligamento. Gestão e Planejamento, 15, 410-427. Recuperado em www.revistas.unifacs.br/index.php/rgb/article/ view/2737

Veloso, E., Dutra, J., Fischer, A., Pimentel, J., Silva, R., \& Amorim, W. (2011). Gestão de carreiras e crescimento profissional. Revista Brasileira de Orientação Profissional, 12, 61-72. Recuperado em http:// pepsic.bvsalud.org/scielo.php?pid=S1679$-33902011000100008 \&$ script $=$ sci_arttext

Wright, T., \& Bonett, D. (2002). The moderating effects of employee tenure on the relation between organizational commitment and job performance: A meta-analysis. Journal of Applied Psychology, 87(6), 1183-1190. doi:10.1037//0021-9010.87.6.1183

Recebido: $23 / 10 / 2015$

$1^{a}$ revisão: $28 / 01 / 2016$

Aceite final: 21/02/2016 